\title{
A Huge Mass of the Duodenum with Upper Gastrointestinal Bleeding
}

\author{
Ju Ho Lee, Hee Seok Moon
}

Division of Gastroenterology, Department of Internal Medicine, Chungnam National University Hospital, Daejeon, Korea

Question: A 40-year-old woman presented with recent epigastric soreness and an episode of melena that started about 2 weeks before. Her past medical history included a left ovarian cyst and uterine myomectomy 1 year before. She initially visited to the local hospital, and a large duodenal mass was found in esophagogastroduodenoscopy (EGD). Then, she was referred to our hospital for further evaluation and treatment of the duodenal lesion. EGD showed a large, solid, lobulated mass with a wide base occupying most of the lumen at the second and third portions of the duodenum without involving the ampulla of Vater (Fig. 1). Spontaneous bleeding was found on the tip of the mass. Abdominal computed tomography revealed an about $5 \times 3 \mathrm{~cm}$-sized, large wide mass with inner small cystic components in the duodenum (Fig. 2).

What is the most likely diagnosis?

Answer: The patient underwent a surgical resection for the duodenal mass. Grossly, the mass was a large polypoid mass measuring $5.5 \times 3.7 \mathrm{~cm}$ in size. Microscopically, the mass consisted of lobules of proliferating Brunner's glands separated by thin fibromuscular bundles beneath the normal duodenal mucosa (Fig. 3). Therefore, the patient was diagnosed with Brunner gland hamartoma of the duodenum.

Brunner's glands are predominantly located in the deep mucosa and submucosa of the proximal duodenum, and decreases in the distal duodenum. They protect the duo- denum from gastric acid by secreting urogastrone, pepsinogen, and mucus. Most Brunner's gland hamartomas are benign, and the incidence of malignancy in Brunner's gland hamartomas is very rare. ${ }^{1}$ The pathophysiology of Brunner's gland hamartoma is not well known yet. Chronic inflammation by excessive secretion of gastric acid, Helicobacter pylori infection, uremia, and pancreatitis are known factors that may cause Brunner's hamartoma. Most patients with Brunner's gland hamartoma are asympto-

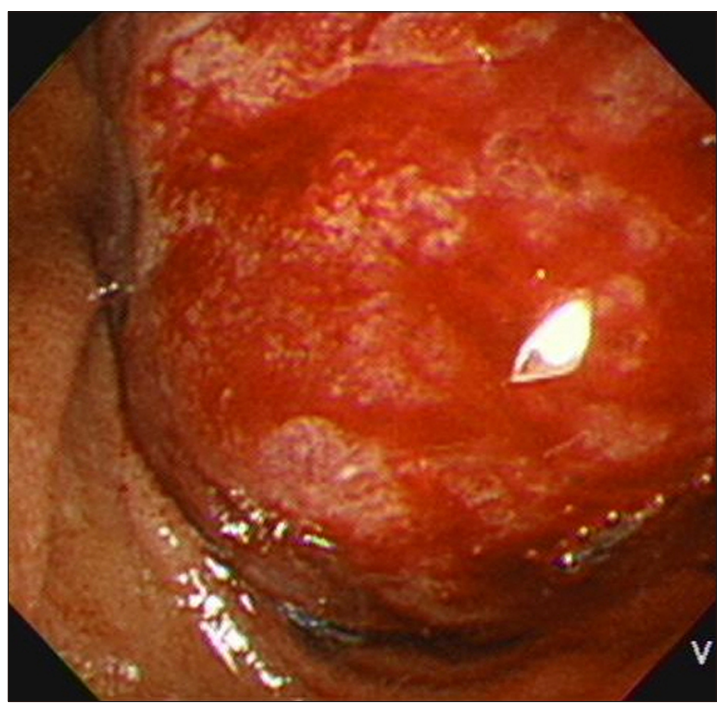

Fig. 1. Esophagogastroduodenoscopy shows a large, pedunculated mass, causing near-total obstruction of the duodenum. The lesion is a solid tumor with a red, lobulated surface and wide base, and spontaneous bleeding is seen. It is located at the opposite side of the ampulla of Vater in the second and third portions of the duodenum.

Received: May 21, 2018 Revised: June 18, 2018 Accepted: June 20, 2018

Corresponding author: Hee Seok Moon

Division of Gastroenterology, Department of Internal Medicine, Chungnam National University Hospital, 266 Munhwa-ro, Jung-gu, Daejeon 35015, Korea Tel: +82-42-280-7164, Fax: +82-42-253-3287, E-mail: mhs1357@cnuh.co.kr (1) ORCID: https://orcid.org/0000-0002-8806-2163

Copyright $\odot 2018$ Korean College of Helicobacter and Upper Gastrointestinal Research

() The Korean Journal of Helicobacter and Upper Gastrointestinal Research is an Open-Access Journal. All articles are distributed under the terms of the Creative Commons Attribution Non-Commercial License (http://creativecommons.org/licenses/by-nc/4.0) which permits unrestricted non-commercial use, distribution, and reproduction in any medium, provided the original work is properly cited. 

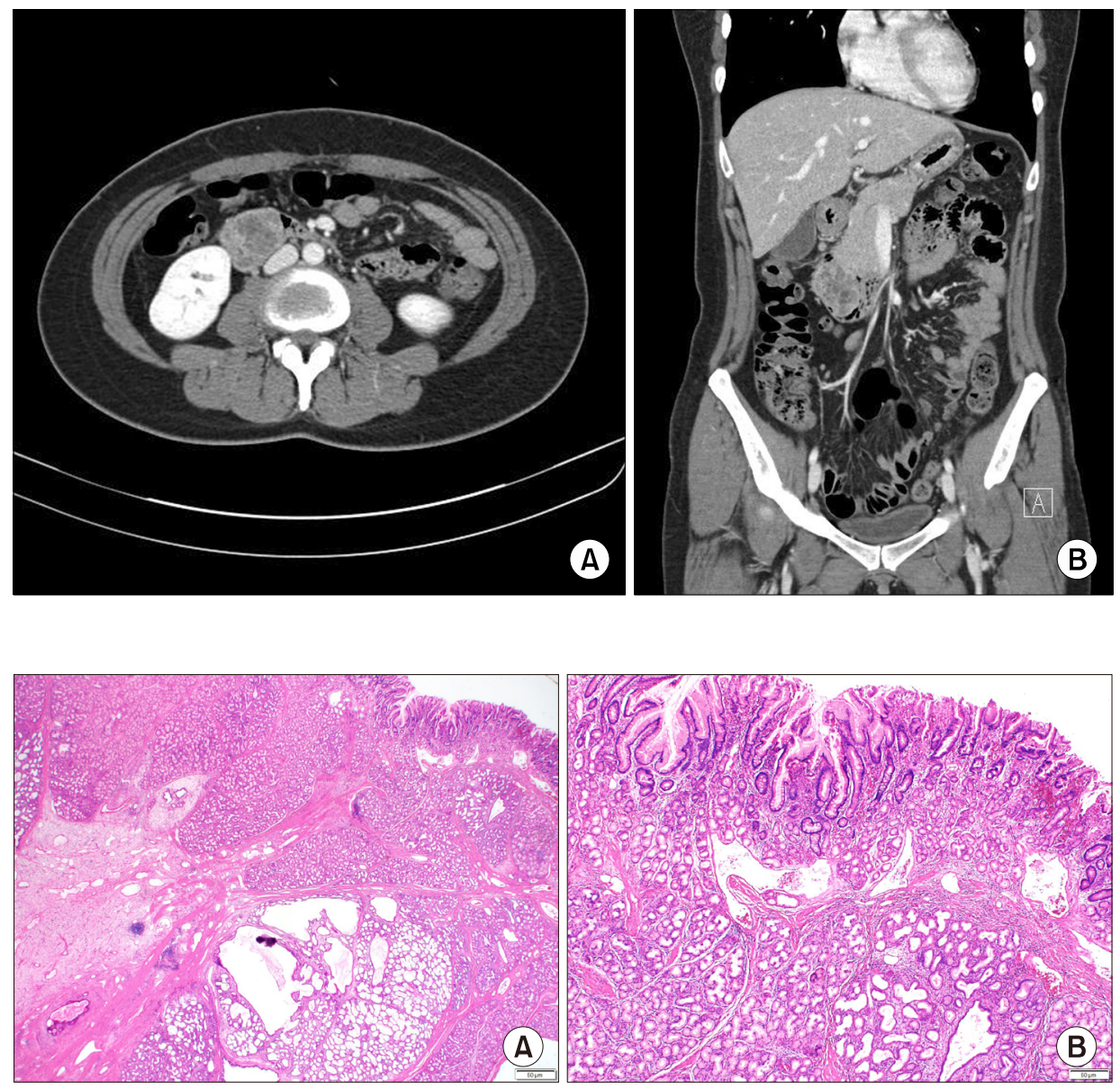

Fig. 2. Contrast-enhanced transverse (A) and sagittal computed tomography (B) shows a pedunculated mass located at the periampullary area in the second and third portions of the duodenum. The lesion is a $5 \times 3-\mathrm{cm}$ sized large mass, occupying the duodenum almost completely and has small cystic components. Signs of tumor invasion and abnormal enlarged lymph nodes are not seen.

Fig. 3. Microscopic findings of the tumor. (A) Low-power view showing lobules of proliferating Brunner's glands separated by thin fibromuscular bundles beneath the normal duodenal mucosa (H\&E, ×12.5). (B) High-power view showing normally appearing Brunner's glands with multifocal cystic dilatations $(\mathrm{H} \& \mathrm{E}, \times 40)$. matic, but sometimes present symptoms such as epigastric pain, gastrointestinal bleeding, obstruction, or intussusception according to the size and location of Brunner's gland hamartoma. Rarely, pancreatitis, obstructive jaundice, or biliary fistula may occur. ${ }^{2}$

Brunner's gland hamartomas are usually found incidentally during regular health check-up EGD. On EGD, Brunner's gland hamartomas appear as reddish, subepithelial lesions with sessile or pedunculated shape in the proximal part of the duodenum. On endoscopic ultrasonography, they usually appear as solid or cystic masses with unclear borders in the deep mucosal and/or submucosal layers. ${ }^{3}$ Treatment are decided according to the size, shape, and severity of symptoms.

Resection is generally performed in symptomatic cases. Endoscopic resection is the preferred treatment modality for small-sized or pedunculated lesions, ${ }^{4}$ but if the size is larger than $2 \mathrm{~cm}$, or if endoscopic resection fails, espe- cially in the case of suspicion of malignant change, surgical resection is favorable.

\section{REFERENCES}

1. Lim W, Kim GH, Park DY. Adenocarcinoma arising from Brunner's gland hyperplasia. Korean J Helicobacter Up Gastrointest Res 2016;16:107-110.

2. Park BJ, Kim MJ, Lee JH, Park SS, Sung DJ, Cho SB. Cystic Brunner's gland hamartoma in the duodenum: a case report. World J Gastroenterol 2009;15:4980-4983.

3. Hizawa K, Iwai K, Esaki M, et al. Endosonographic features of Brunner's gland hamartomas which were subsequently resected endoscopically. Endoscopy 2002;34:956-958.

4. Song EM, Shim KN, Kang HW, et al. A case of Brunner's gland adenoma presenting as large polyp with stalk. Korean J Helicobacter Up Gastrointest Res 2012;12:47-49.

5. Lee WC, Yang HW, Lee YJ, et al. Brunner's gland hyperplasia: treatment of severe diffuse nodular hyperplasia mimicking a malignancy on pancreatic-duodenal area. J Korean Med Sci 2008;23:540-543. 